\title{
Oficina educativa Atenção Centrada no Paciente: Um relato de experiência
}

\author{
Educational workshop Patient Centered Care: An experience report \\ Taller educativo Atención Centrada en el Paciente: Informe de experiência
}

Recebido: 14/04/2021 | Revisado: 21/04/2021 | Aceito: 25/04/2021 | Publicado: 10/05/2021

Carine Freitas Galvão Vieira

ORCID: https://orcid.org/0000-0002-8938-7736

Universidade Federal do Maranhão, Brasil

E-mail: carine.fgv@ discente.ufma.br

Sara Fiterman Lima

ORCID: https://orcid.org/0000-0003-0015-3413

Universidade Federal do Maranhão, Brasil

E-mail: sara.fiterman@ufma.br

Julyana Suelen Rodrigues Fonseca

ORCID: https://orcid.org/0000-0002-7777-7681

Universidade Federal do Maranhão, Brasil

E-mail: julyana.fonseca@ discente.ufma.br

Cecílio Soares Rodrigues Braga

ORCID: https://orcid.org/ 0000-0002-2313-7646

Universidade Federal do Maranhão, Brasil E-mail:csrb_8@hotmail.com

Ramon Souza Costa

ORCID: https://orcid.org/ 0000-0002-9793-551X

Universidade Federal do Maranhão, Brasil

E-mail: ramon.costa@discente.ufma.br

João de Jesus Oliveira Junior

ORCID: https://orcid.org/0000-0002-3259-6131

Universidade Federal do Maranhão, Brasil E-mail: joaodejota@gmail.com

Amanda Namíbia Pereira Pasklan

ORCID: https://orcid.org/0000-0001-7193-4861

Universidade Federal do Maranhão, Brasil E-mail: amanda.namibia@ufma.br

Mayara Cristina Pinto da Silva

ORCID: https://orcid.org/0000-0002-1868-6931

Universidade Federal do Maranhão, Brasil

E-mail: mayara.silva@ufma.br

\begin{abstract}
Resumo
Objetivo: Relatar a experiência vivenciada por participantes da nona versão do PET-Saúde, em uma oficina educativa sobre competência colaborativa- Atenção Centrada no Paciente/Usuário. Método: Trata-se de um estudo descritivo, do tipo relato de experiência, realizado por integrantes do projeto de extensão PET-Saúde Interprofissionalidade, da Universidade Federal do Maranhão (UFMA), Campus Pinheiro. A oficina educativa intitulada "Competências Colaborativas: Atenção Centrada no Paciente/Usuário", foi ofertada para 44 participantes, incluindo discentes, docentes e preceptores. Aplicaram-se instrumentos de avaliação pós-testes imediato e tardio (após cinco meses). Resultados: Verificou-se que a oficina alcançou os resultados esperados, haja visto que no questionário tardio os participantes declararam que obtiveram e aplicaram conhecimentos de modo satisfatório a respeito da competência trabalhada. Alegaram, também, melhora na relação entre profissionais e pacientes. Conclusão: Acredita-se que os resultados da experiência foram satisfatórios. Os conhecimentos trabalhados na Oficina permaneceram presentes nos entrevistados mesmo após cinco meses da sua realização. Além disso, a atividade permitiu uma análise crítica da realidade entre os participantes, incentivando o desejo de promover e demonstrar os conhecimentos adquiridos sobre educação interprofissional e de realizar uma prática mais colaborativa centrada no paciente/usuário, família e comunidade.
\end{abstract}

Palavras-chave: Relações interprofissionais; Educação em saúde; Comportamento cooperativo.

\begin{abstract}
Objective: The article seeks to report the experience lived by participants of the ninth version of PET-Saúde, in a educational workshop on collaborative competence - Patient / User Centered Care. Method: This is a descriptive study, an experience report, carried out by members of the project PET-Saúde Interprofissionalidade an extension program, at Federal University of Maranhão (UFMA), Campus Pinheiro. The educational workshop entitled "Collaborative Competences: Attention Centered on Patient / User", was offered to 44 participants, including
\end{abstract}


students, teachers and preceptors. Immediate and late (after five months) post-test assessment instruments were applied. Results: It was found that the workshop achieved the expected results, given that in the late questionnaire the participants stated that they obtained and applied knowledge in a satisfactory manner regarding competence worked on. They also claimed an improvement in the relationship between professionals and patients. Conclusion: It is believed that the results of the experiment were satisfactory. Knowledge worked at the Workshop remained present in the interviewees even after five months of their achievement. In addition, the activity allowed a critical analysis of the reality among the participants, encouraging the desire to promote and demonstrate the knowledge acquired on education interprofessional and to carry out a more collaborative practice centered on the patient / user, family and community

Keywords: Interprofessional relations; Health education; Cooperative behavior.

\section{Resumen}

Objetivo: Informar la experiencia vivida por los participantes de la novena versión de PET-Saúde, en un taller educativo sobre competencia colaborativa - Atención Centrada en Paciente / Usuario. Método: Se trata de un estudio descriptivo, un relato de experiencia, realizado por miembros del proyecto de extensión de Interprofesionalidad PETSaúde, en la Universidad Federal de Maranhão (UFMA), Campus Pinheiro. El taller educativo titulado "Habilidades colaborativas: atención centrada en el paciente / usuario", se ofreció a 44 participantes, entre estudiantes, docentes y preceptores. Se aplicaron instrumentos de evaluación posprueba inmediata y tardía (después de cinco meses). Resultados: Se constató que el taller logró los resultados esperados, dado que en el cuestionario tardío los participantes declararon haber obtenido y aplicado conocimientos de manera satisfactoria sobre la competencia trabajada. También afirmaron una mejora en la relación entre profesionales y pacientes. Conclusión: Se cree que los resultados del experimento fueron satisfactorios. Los conocimientos trabajados en el Taller permanecieron presentes en los entrevistados incluso después de cinco meses de su finalización. Además, la actividad permitió un análisis crítico de la realidad entre los participantes, fomentando el deseo de promover y demostrar los conocimientos adquiridos en educación interprofesional y realizar una práctica más colaborativa centrada en el paciente / usuario, familia y comunidad.

Palabras clave: Relaciones interprofesionales; Educación para la salud; Comportamiento cooperativo.

\section{Introdução}

O conceito amplo de saúde é relativamente recente. Essa concepção surge com o desenvolvimento da Saúde Pública no início do século XX e é reafirmado pela posição da Organização Mundial da Saúde (OMS) que define saúde como um estado de completo bem-estar físico, mental e social. Para permitir um trabalho coeso e integrado entre as mais diferentes áreas da saúde foi necessário desenvolver um modelo de educação que integrasse desde a graduação as mais distintas profissões da saúde, surge então a educação interprofissional (EIP) (Lima et al, 2020).

A Educação Interprofissional (EIP), por sua vez, acontece quando alunos ou membros de duas ou mais profissões aprendem com, a partir e sobre o outro para melhorar a colaboração e a qualidade do cuidado. Se configura como estratégia para o estímulo à formação coerente com as necessidades de fortalecimento do SUS. (Centre for the Advancement of Interprofessional Education) [Caipe] (2013).

A história de construção e consolidação do SUS é marcada por debates sobre a integralidade do cuidado, e as necessidades sociais e de saúde da população, a partir de uma concepção ampliada de saúde (Costa \& Costa, 2016).

Ao longo da história, os profissionais de saúde têm recebido formação que enfatiza o desenvolvimento de competências específicas, legitimando o que a literatura define como silos profissionais ou tribalismo das profissões. Esse modelo de formação sustenta práticas fortemente fragmentadas, com exposição do usuário a atos duplicados, e leva ao retrabalho dos profissionais de saúde, aumentando os riscos de erros e custos em saúde (Freire Filho et al., 2019). Entretanto, existe uma demanda que transcende os fazeres individualizados de cada profissão e projeta a valorização da equipe, na qual o profissional não renuncia à sua especificidade, mas valoriza o trabalho cooperativo em ações direcionadas à população (Casanova et al., 2018).

Faz-se necessário a compreensão que o Brasil vive um momento de transição epidemiológica denominado tripla carga de doenças. Essa transição é marcada pela existência de comorbidades de cunho infecto parasitária, doenças crônico degenerativas e causas externas, tais como a violência urbana e acidentes de trânsito (Mendes,2010). Para superar esse período 
crítico observa-se que o modelo antigo de fragmentação das áreas da saúde não é capaz de suprir todas as demandas, cada vez mais complexos, do serviço de saúde. Sendo assim, o sistema de saúde como um todo clama por um novo perfil profissional que consiga prover um atendimento mais integral e que saiba atuar de forma conjunta com os mais diversos profissionais, para enfim promover uma melhora efetiva em todo sistema de saúde (Matuda,2013).

Nesse contexto, emerge a potência da EIP como movimento de mudança na lógica de formação em saúde. Ela potencializa o cuidado integral em saúde juntamente com os princípios e diretrizes do SUS, melhorando assim o sistema de saúde. No contexto histórico, a EIP é um modelo de intervenção que possui maior capacidade de gerar uma maior qualidade da atenção em saúde, justamente por facilitar trabalho em equipe entre diferentes profissões (Batista et al., 2018; Costa \& Costa, 2016; Organização Mundial da Saúde [OMS] (2010).

Assim, a prática interprofissional colaborativa tem papel crucial para a atenção centrada no paciente, pois as ações em saúde estão voltadas às necessidades de usuários, das famílias e da comunidade. Torna-se necessário que a integralidade da assistência englobe ações interprofissionais, interdisciplinares e intersetoriais de atenção à saúde (Agreli et al., 2016).

Destaca-se que a EIP tem grande possibilidade de promover a consolidação de sistemas e os serviços de saúde, melhorando de forma significativa a eficácia da atenção básica de saúde. Além disso, promovem a redução dos custos com sistema de saúde, eliminação de procedimentos e condutas sem necessidade que elevam a segurança do paciente, e diminuição dos erros dos profissionais de saúde (Reeves et al., 2009).

Acredita-se que a proposta da EIP é reformular o contexto atual de formação e prática profissional. Para isso, a interação entre as demais profissões torna-se crucial, por contribuir com a compreensão do papel que cada profissional exerce no sistema de saúde. Tais relações são fundamentais para o desenvolvimento das competências colaborativas, que melhoram a relação entre a equipe, favorece a comunicação entre os membros, cooperando para a humanização, qualidade e segurança do cuidado (Lima et al., 2020).

Acrescenta-se, diante do exposto, que a prática das competências colaborativas interprofissionais favorece a formação de profissionais mais preparados para o trabalho em equipe, para a resolução de conflitos, mais aptos a enfrentarem situações impostas, e capazes de atender o usuário com qualidade, eficiência e resolutividade, conforme os referenciais nacionais e internacionais de saúde (Fonsêca, 2018).

Torna-se essencial investir em ações que fortaleçam tais práticas, visto que organizam o trabalho e fortalecem a colaboração interprofissional. As atividades elaboradas através de oficinas educativas, representam uma excelente proposta para a educação interprofissional colaborativa. Pois oferecem troca de conhecimentos e contribuição para uma aprendizagem efetiva com olhar diferenciado, os quais não teriam caso trabalhassem de maneira isolada (Griggio et al., 2018). Desse modo, o presente artigo teve como objetivo relatar a experiência vivenciada por participantes da nona versão do PET-Saúde em uma oficina educativa sobre a Atenção Centrada no Paciente.

\section{Metodologia}

Trata-se de um estudo descritivo, quantitativo, do tipo relato de experiência, realizado por integrantes do projeto de extensão PET-Saúde Interprofissionalidade, da Universidade Federal do Maranhão (UFMA), Campus Pinheiro. O projeto PET-Saúde Interprofissionalidade é um dos ramos de uma política de implementação da EIP no Brasil, ele é promovido em uma ação conjunta do Ministério da Saúde, do Ministério da Educação e a Rede Brasileira de Educação e Trabalho Interprofissional em Saúde. Nesse contexto, temos que o PET-Saúde é uma peça indispensável para a adoção das competências e habilidades necessárias para a promoção do trabalho em equipe de maneira coesa e interprofissional (Silva et al., 2018).

Como parte desse projeto, e objeto de relato, foi ofertada a oficina educativa intitulada "Competências Colaborativas: Atenção Centrada no Paciente" que contou com a presença de 44 participantes. Com duração de quatro horas, a oficina ocorreu 
em um encontro presencial no mês de janeiro de 2020. Participaram acadêmicos dos cursos de Educação Física, Enfermagem e Medicina, docentes tutores e profissionais da área de saúde preceptores. Foi-lhes garantido, para isso, o anonimato e amparo ético mediante assinatura de Termo de Consentimento Livre e Esclarecido (TCLE). O número do parecer consubstanciado CEP (Comitê de ética em pesquisa) é 3.938.591 e CAAE é: 29652520.0.0000.5087.

Como estratégias educacionais, utilizou-se apresentação oral temática com recurso de projeção de imagem sobre "Competências Colaborativas: Atenção Centrada no Paciente" e reprodução do vídeo DRC - Atendimento interdisciplinar ao paciente - parte 1 e parte 2. Em seguida, executou-se dinâmicas relacionadas a apresentação oral da competência esplanada. Promoveu-se a oficina com a participação dos integrantes do programa PET-SAÚDE Interprofissionalidade, proporcionando aos presentes um momento de construção e aperfeiçoamento dos conhecimentos acerca da educação interprofissional. A temática abordada foi a "Atenção Centrada no Paciente", que integra o conjunto de competências colaborativas para a efetividade da EIP.

Trabalhou-se sob a perspectiva teórico-prática, transcorrendo pelos seguintes pontos: reconhecer a singularidade do paciente como centro dos cuidados; estimular a participação do usuário e de seus familiares na tomada de decisão dos cuidados. Tais pontos serviram de guia para a programação da oficina, que utilizou recursos metodológicos para proporcionar um melhor aproveitamento.

A aula foi mediada por dois integrantes discentes do grupo tutorial de Imunização, onde reforçaram que a ACP é parte imprescindível da prática interprofissional colaborativa, sendo que esta possui três pilares fundamentais: perspectiva ampliada do cuidado à saúde, que visa atender de forma integral as carências de saúde do indivíduo, familiares e do coletivo; participação no cuidado, que fortalece a autossuficiência do indivíduo, contribuindo para o autocuidado; relação profissional e paciente, que considera as particularidades do indivíduo enquanto constituintes do seu próprio processo de cuidado (Agreli et al., 2016).

Posterior ao momento teórico, dois vídeos, no qual abordaram o atendimento ao paciente. Após a apresentação, os participantes iniciaram uma discussão, elencando vários pontos e comentando sobre a diferença entre os termos multidisciplinaridade, interdisciplinaridade e interprofissionalidade; juntos, articularam como seria o modo mais adequado de prestar a assistência, reforçando os princípios da Educação Interprofissional. Citou-se a importância da horizontalidade do cuidado, que este não deve mais obedecer a níveis hierárquicos, pois esta é uma prática equivocada e contribui para a fragmentação do cuidado.

Optou-se por formar grupos, para que os partícipes pudessem compreender sobre os diferentes tipos de pacientes e suas singularidades, onde foram entregues casos clínicos para que os participantes pudessem compreender o tipo de paciente no qual estavam lidando e assim montar uma linha de cuidado adequada individualizada, respeitando as particularidades do usuário; utilizou-se essa estratégia para que pudessem simular um atendimento interprofissional com foco para o paciente.

Ao término da oficina, os participantes foram convidados a expressar seu feedback imediato e tardio por meio de questionário eletrônico, composto por perguntas objetivas e discursivas. Foram registrados os contatos de e-mail e telefone a fim de coletar feedback tardio, com uma busca ativa dos participantes cinco meses após a oficina. As respostas obtidas via questionários foram inseridas em um banco de dados e submetidas ao programa estatístico Epi Info, versão 7.2. A análise foi realizada mediante abordagem descritiva. Foram feitas as distribuições de frequências absoluta e relativa para variáveis categóricas.

\section{Resultados}

As atividades da oficina iniciaram-se com um momento de compartilhamento de experiências entre profissionais e acadêmicos, onde puderam discutir a respeito da Atenção Centrada no Paciente e familiarizar-se com o tema. No primeiro 
momento, houve uma aula expositiva dialogada sobre o conceito de Atenção Centrada no Paciente, onde foram considerados os conhecimentos prévios dos presentes em relação ao tema.

Foi observado que ao longo de sua participação no projeto, os integrantes estão cada vez mais inteirados sobre a abordagem interprofissional e isso repercute positivamente em seus ambientes de trabalho e acadêmico. É notório que há cada vez mais uma aproximação entre as diferentes profissões, resultando em reconhecimento dos diferentes papéis profissionais no serviço de saúde.

Através de uma avaliação pós oficina, constatou-se que esse momento foi de grande valia para os presentes. Os impactos autorreferidos são de melhorias na atenção ao paciente, família e comunidade; contribuindo para uma ótica diferenciada pelos profissionais de saúde, que passaram a tratar os seus pacientes de forma integral, considerando todas as suas especificidades e tornando-o responsável pelo seu cuidado. Contribuiu para uma formação discente de qualidade, sendo que estes estão tendo acesso a uma formação diferenciada, o que, certamente, resultará em profissionais mais hábeis ao trabalho em equipe.

Foi disponibilizado um questionário online aos presentes para avaliar a repercussão da oficina, se esta teve pontos positivos ou negativos (Tabela 1).

Tabela 1. Análise do questionário realizado logo após a oficina ofertada pelo PET-Saúde. Pinheiro, MA, 2020.

\begin{tabular}{|c|c|c|}
\hline Variável & $\mathbf{n}$ & $\%$ \\
\hline \multicolumn{3}{|l|}{ Vínculo Profissional } \\
\hline Município & 4 & 19,05 \\
\hline Universidade & 17 & 80,95 \\
\hline \multicolumn{3}{|l|}{ Como você avalia a nossa oficina } \\
\hline 8 & 1 & 4,76 \\
\hline 9 & 7 & 33,34 \\
\hline 10 & 13 & 61,90 \\
\hline \multicolumn{3}{|c|}{$\begin{array}{l}\text { Você já havia discutido esse assunto durante sua } \\
\text { formação }\end{array}$} \\
\hline Sim & 11 & 52,38 \\
\hline Não & 6 & 28,57 \\
\hline Talvez & 4 & 19,05 \\
\hline \multicolumn{3}{|c|}{$\begin{array}{l}\text { Pontue seu aprendizado sobre atenção centrada no } \\
\text { paciente após a oficina }\end{array}$} \\
\hline 8 & 3 & 14,28 \\
\hline 9 & 9 & 42,86 \\
\hline 10 & 9 & 42,86 \\
\hline
\end{tabular}

Fonte: Autores.

Quando perguntados sobre o esclarecimento a respeito dos conceitos abordados durante a nossa discussão como: equipe multiprofissional, equipe interdisciplinar e equipe interprofissional, todos os participantes alegaram ter compreendido e aprendido a diferenciar esses conceitos.

Ademais, os participantes concordaram que a formação no PET-Saúde Interprofissionalidade foi indispensável para essa compreensão. Após cerca de cinco meses aplicou-se um segundo questionário a fim de avaliar como foi a fixação do conteúdo exposto (Tabela 2). 
Tabela 2. Análise do questionário realizado cinco meses após a oficina ofertada pelo PET-Saúde. Pinheiro, MA, 2020.

\begin{tabular}{lll}
\hline Variável & $\mathbf{n}$ & $\%$ \\
\hline Você é? & 16 & 69,57 \\
\hline Aluno da Graduação & 7 & 30,43 \\
Profissional de Saúde & & 8,70 \\
Você pode observar a participação do paciente na tomada & 2 & 33,34 \\
de decisões? & 7 & 91,30 \\
\hline $\begin{array}{l}\text { Não, o profissional é o responsável por determinar o cuidado. } \\
\text { Sim, há o envolvimento do paciente no seu processo de }\end{array}$ & 21 & 4,35 \\
cuidado. & & 78,26 \\
Você acredita que o princípio da integralidade da atenção é & 1 & 17,39 \\
devidamente aplicado no SUS? & 18 & \\
\hline $\begin{array}{l}\text { Sim, é devidamente aplicado na prática interprofissional. } \\
\text { Sim, mas ainda é necessário aprimorar os conhecimentos dos }\end{array}$ & & \\
profissionais sobre o tema. & 4 & \\
Não, não se aplica o princípio da integralidade da atenção. & & \\
\hline
\end{tabular}

Fonte: Autores.

Além dos questionamentos abordados na tabela acima (Tabela 2), todos os participantes da oficina que aceitaram participar da pesquisa alegaram que a oficina de competências colaborativas contribuiu para uma melhora da relação entre profissionais e paciente. Os participantes também concordaram que a oficina ajudou na capacitação do profissional sobre atenção centrada no paciente, deixando claro que o objetivo principal da oficina, passar um conteúdo que pode ser aplicado na prática foi alcançado.

Além disso, quando perguntados se acreditam que o tema de atenção centrada no paciente deveria ser implementado nas matrizes curriculares do ensino superior a resposta foi unânime, todos os participantes concordaram que é um tema que deve ser abordado durante a graduação.

\section{Discussão}

O desenvolvimento de práticas colaborativas de atenção centrada no paciente promove um impacto relevante na qualidade do serviço de saúde e a prática interprofissional, por sua vez, tem um papel de destaque na aplicabilidade dessa competência (REIP, 2019). Dentro desse processo o programa PET- Saúde Interprofissionalidade se mostra como parte indispensável para obtenção e aplicação desses conceitos. Ademais, para que exista um desenvolvimento teórico-prático dos conceitos das competências colaborativas devemos desenvolver um ambiente de debate, participação e interação ativa no processo de construção dos saberes, desse modo a realização de oficinas se mostram como uma ferramenta pedagógica significativa de aprendizado (Sargeant, 2009).

$\mathrm{Na}$ avaliação da oficina perguntou-se a os participantes se sua prática profissional foi alterada após a oficina. Dentre as respostas pode-se perceber mudanças na forma de observar o paciente com atenção em fatores como valores, crenças e costumes; na forma de incluir o paciente em seu processo de cuidado negociando com ele a melhor estratégia; e houve uma atenção maior nos outros profissionais membros da equipe. Dentre os empecilhos que dificultam a implantação dessa competência as respostas se resumiram na falta de interesse de outros profissionais da equipe o que demonstra uma necessidade crescente em projetos de educação permanente em saúde que estimulem a intencionalidade da prática e 
aprendizado profissional. Tais achados corroboram com estudos similares, nos quais a intervenção de educação interprofissional propiciou ganhos e reiterou desafios, a exemplo da fragilidade na formação profissional e sua reverberação na concretização do trabalho em equipe interprofissional (Griggio et al, 2020; Artaza et al, 2020). Nesse interim, a fim de mitigar essas falhas, emerge a necessidade de reformular as grades curriculares priorizando o aprendizado colaborativo (SandovalBarrientos et al, 2019).

Destaca-se que a oficina atingiu os objetivos esperados haja visto que, na segunda aplicação do questionário, cinco meses após a oficina, os conhecimentos a respeito da atenção centrada no paciente se mantiveram. Intervenções como essa agregam melhorias na apreensão de conhecimentos comuns e específicos, mesmo que sua aplicabilidade seja dependente da inserção na rotina dos serviços em saúde (Agreli et al, 2019). Quando se perguntou a respeito de como deve ser o cuidado centrado no paciente as respostas obtidas foram: deve ser um cuidado em que o paciente deve ser ouvido e levado em consideração as suas necessidades tanto a nível individual como em grupo; e pensado pelo maior número de profissionais que possam auxiliar alguma demanda do paciente, onde eles intercambiam informações e constroem conjuntamente entre eles e com o paciente um plano de cuidados. Com isso, ratifica-se o empoderamento em saúde de indivíduos, famílias e comunidades proporcionado pela EIP (Artaza et al, 2020).

Desse modo, percebeu-se a importância da educação interprofissional que consiste em duas ou mais profissões que aprendem sobre os outros, com os outros e entre si para a efetiva colaboração e melhora dos resultados na saúde (OMS, 2010). Diante da demanda exigida pelo mercado de trabalho, em especial pelos princípios e diretrizes do Sistema Único de Saúde (SUS), a EIP está cada vez mais no âmbito das discussões. Isso ocorre pois observou-se um anseio urgente para a reorganização dos serviços de saúde, na qual observa-se um cuidado mais efetivo, integral, resolutivo, reduzindo os custos e os erros e desse modo, centrado no paciente (Almeida et al., 2019).

Como limitações do estudo podemos citar que não houve uma avaliação dos conhecimentos prévios dos participantes impossibilitando a distinção plena entre conhecimentos adquiridos na oficina e vivências prévias. No entanto, tal fato não prejudicou a qualidade dos dados obtidos e sua colaboração para avaliar a importância da educação interprofissional para a garantia da atenção centrada ao paciente/usuário.

\section{Conclusão}

Constata-se que a realização da oficina possibilitou, aos envolvidos, momentos ricos de discussões e aprendizados, com base na competência colaborativa-Atenção Centrada no Paciente/Usuário. A oficina educativa serviu de reflexão para análise da nossa realidade no atendimento ao paciente/usuário e despertou a importância da intencionalidade para a aprendizagem interprofissional.

Percebe-se que o PET-Saúde com a realização da oficina educativa, trouxe uma forte contribuição para a formação dos extensionistas, pois possibilitou o aprendizado a partir da participação da oficina frente às experiências compartilhadas pelos preceptores e tutores.

Acredita-se que os resultados da experiência foram satisfatórios, uma vez que essa reflexão permitiu a ampliação da capacidade de análise crítica da realidade, somada ao desejo futuro de promover e demonstrar os benefícios da educação interprofissional para a prática colaborativa centrada no paciente/usuário, família e comunidade.

No entanto, acreditamos que mais estudos e avaliações quanto a presença dessas competências entre profissionais da atenção primária seja necessária. Para tanto, faz-se necessário tanto que que mais grupos promovam a educação interprofissional dentro e fora dos espaços acadêmicos quanto que o serviço de saúde estimule o desenvolvimento das habilidades e competências relacionadas à interprofissionalidade. Ademais, mostra-se fundamental que seja desenvolvido meios validados para promover uma avaliação da introdução prática de tais conceitos a fim de promover a avaliação uniforme 
dessas práticas.

\section{Referências}

Agreli, H. F., Peduzzi, M., \& Silva, M. C. (2016). Atenção centrada no paciente na prática interprofissional colaborativa. Interface - Comunicação, Saúde, Educação, 20(59), 905-916. https://doi.org/10.1590/1807-57622015.0511

Agreli, H. F., Peduzzi, M., Silva, M. C., Mascarelle, R. C. V., Espinoza, P. (2019). Efeito da educação interprofissional no trabalho em equipe e no conhecimento do manejo de condições crônicas. Revista Latino-Americana de Enfermagem, 27, e3203. https://dx.doi.org/10.1590/1518-8345.3095.3203

Almeida, Rodrigo Guimarães dos Santos, Teston, Elen Ferraz, \& Medeiros, Arthur de Almeida. (2019). A interface entre o PET-Saúde/Interprofissionalidade e a Política Nacional de Educação Permanente em Saúde. Saúde em Debate, 43(1), 97-105. 16, 2019.https://dx.doi.org/10.1590/0103-11042019s108

Artaza, O., Santacruz, J., Girard, J., Alvarez, D., Barría, S., Tetelboin, C., Tomasina, F., Medina, A. (2020). Formación de recursos humanos para la salud universal: acciones estratégicas desde las instituciones académicas. Rev Panam Salud Publica, 44, 1-5. https://doi.org/10.26633/RPSP.2020.83

Batista, N. A., Rossit, R. A. S., Batista, S. H. S. S., Silva, C. C. B., Uchôa-Figueiredo, L. da R., Poletto, P. R. (2018). Educação interprofissional na formação em Saúde: A experiência da Universidade Federal de São Paulo, campus Baixada Santista, Santos, Brasil. Interface - Comunicação, Saúde, Educação, 22, 1705-1715. https://doi.org/10.1590/1807-57622017.0693

Casanova, I. A., Batista, N. A., \& Moreno, L. R. (2018). A Educação Interprofissional e a prática compartilhada em programas de residência multiprofissional em Saúde. Interface - Comunicação, Saúde, Educação, 22, 1325-1337. https://doi.org/10.1590/1807-57622017.0186

Centre for the Advancement of Interprofessional Education [Caipe] (2013). Defining IPE [internet]. Recuperado em 22 de janeiro de 2021, de https://www.caipe.org/resources/publications/caipe-publications/barr-h-low-h-2013-introducing-interprofessional-education-13th-november-2016

Costa, M. V. da, \& Costa, M. V. da. (2016). The interprofessional education in Brazilian context: Some reflections. Interface - Comunicação, Saúde, Educação, 20(56), 197-198. https://doi.org/10.1590/1807-57622015.0311

Fonsêca, R. M. D. (2018). Educação interprofissional em saúde e o desenvolvimento de competências colaborativas na formação em enfermagem e medicina (Master's thesis, Brasil).

Freire Filho, J. R., Silva, C. B. G., Costa, M. V. da, Forster, A. C., Freire Filho, J. R., Silva, C. B. G., Costa, M. V. da, \& Forster, A. C. (2019). Educação Interprofissional nas políticas de reorientação da formação profissional em saúde no Brasil. Saúde em Debate, 43, 86-96. https://doi.org/10.1590/010311042019 s 107

Griggio, A. P., Mininel, V. A., \& Silva, J. A. M. D. (2018). Planejamento de uma atividade de educação interprofissional para as profissões da Saúde. Interface-Comunicação, Saúde, Educação, 22, 1799-1809. https://doi.org/10.1590/1807-57622017.0831

Griggio, A. P., Silva, J. A. M. da, Rossit, R. A. S., Mieiro, D. B., Miranda, F. M. de, Mininel, V. A. (2020). Análise de uma atividade de educação interprofissional na área de saúde do trabalhador. Revista Latino-Americana de Enfermagem, 28, e3247. https://dx.doi.org/10.1590/1518-8345.3228.3247

Lima, A. W. S. D., Alves, F. A. P., Linhares, F. M. P., Costa, M. V. D., Coriolano-Marinus, M. W. D. L., \& Lima, L. S. D. (2020). Perception and manifestation of collaborative competencies among undergraduate health students. Revista latino-americana de enfermagem, 28. https://doi.org/10.1590/15188345.3227.3240

Matuda, C. G., Aguiar, D. M. de L., \& Frazão, P. (2013). Cooperação interprofissional e a Reforma Sanitária no Brasil: implicações para o modelo de atenção à saúde. Saúde e Sociedade, 22(1), 173-186. https://doi.org/10.1590/S0104-12902013000100016

Mendes, Eugênio Vilaça. (2010). As redes de atenção à saúde. Ciência \& Saúde Coletiva, 15(5), 2297-2305. https://doi.org/10.1590/S141381232010000500005

Organização Mundial da Saúde. (2010). Marco para a ação em Educação Interprofissional e Prática Colaborativa. OMS. Editora Freelance.

Reeves, S., Goldman, J., \& Zwarenstein, M. (2009). An emerging framework for understanding the nature of interprofessional interventions. Journal of interprofessional care, 23(5), 539-542. 10.1080/13561820903078215

Rede Regional de Educação Interprofissional das Américas e Organização Pan-Americana da Saúde (REIP). (2019). Atenção centrada no paciente na prática interprofissional colaborativa: elementos-chave, estratégias e caminhos a seguir.

Silva, Fernando Antonio Menezes da, Cassiani, Silvia Helena De Bortoli, \& Freire Filho, José Rodrigues. (2018). Interprofessional Health Education in the Region of the Americas. Revista Latino-Americana de Enfermagem, 26, e3013. https://doi.org/10.1590/1518-8345.0000.3013

Sandoval-Barrientos, S., Arntz-Vera, J., Flores-Negrin, C., Trunce-Morales, S., Pérez-Carrasco, A., López-Uribe, J., Velásquez-Scheuch, J. (2019). Propuesta de formación interprofesional en 4 programas de licenciatura de profesionales sanitarios. Educ. med. 20 (2), 25-32. https://doi.org/10.1016/j.edumed.2017.12.006

Sargeant J. (2009). Theories to aid understanding and implementation of interprofessional education. The Journal of continuing education in the health professions, 29(3), 178-184. https://doi.org/10.1002/chp.20033 